\title{
Theoretical Study of Electric Field-Dependent Polaron-type Mobility in Conjugated Polymers
}

\author{
Helena M. G. Correia, Marta M. D. Ramos \\ Physics Department, University of Minho, \\ Campus de Gualtar, 4710-059 Braga, Portugal
}

\begin{abstract}
We have used a self-consistent quantum molecular dynamics approach to calculate the mobility of both positive and negative polaron-type carriers on isolated chains of poly(p-phenylene vinylene) (PPV) and some of its derivatives and the dependence of their mobility on the applied electric field. Our results suggest that polaron-type mobility along most of these polymer chains has a clear dependence on the electric field which is quite different from the result derived for bulk PPV-based materials.
\end{abstract}

\section{Introduction}

The use of conjugated semiconducting polymers as an active material in optoelectronic devices [1, $2]$ is an area of current research interest. It has been recognized that the charge transport properties of these materials are of great importance for the operation and efficiency of such polymer-based devices. Although the conducting properties of an isolated polymer chain are difficult to obtain experimentally, a few attempts have been made experimentally to determine the intra-chain mobilities of positive and negative charge carriers on derivatives of poly(p-phenylene vinylene) (PPV) $[3,4]$. However, the factors that control the charge mobility along these polymer chains are not well understood. For example, it is not clear how the charge-transport properties of isolated chains depend on their internal structure, conjugation length, twisting of conjugated segments as well as on the applied electric field.

The purpose of this paper is to use a theoretical approach to investigate these problems. Since conduction in conjugated polymers is believed to be mediated by charge-induced defects (localized charge dressed by lattice distortion) arising from the strong electron-lattice coupling, we use a selfconsistent quantum molecular dynamics method to calculate quantitatively the mobility of these charged defects, known as polarons, on isolated chains of PPV [5], cyano-PPV (CN-PPV) [6] and dimetoxy-PPV (DMeO-PPV) [7] and the dependence of their mobility on the electric field. The effects of conjugation length and conjugation break on the mobility of positive and negative charged polarons on PPV are also discussed.

\section{Theoretical method}

Because of the strong level of electron-lattice coupling in conjugated polymers, it is necessary to perform self-consistent calculations of electronic wave functions and atomic positions in order to properly study the charge-induced defects in these materials and their mobility along isolated chains. The method used in this work, implemented in the CHEMOS code [8, 9], solves simultaneously the Hartree-Fock equations in the Complete Neglect of Differential Overlap approximation for the electronic structure of an isolated polymer chain and Newton's equations of motion for its atoms, using the forces calculated self-consistently at each time step. When an electric field is applied, the Hamiltonian of the polymer chain gets modified which affects not only the wave functions and energy of the chain but also the force acting on each atom. 


\section{Results and discussion}

When one or two electrons (or holes) are injected into a straight polymer chain with 16 monomer units of PPV, CN-PPV and DMeO-PPV, there is a charge rearrangement of the carbon atoms of the polymer backbone which is accompanied by a change in its dimerisation pattern (defined by the absolute value of the difference between adjacent carbon-carbon bond length at each carbon atom). In the absence of an applied electric field the charge-induced defects of single and double charged chains are localized at the positions shown on the left-hand side of Fig. 1, regardless of the sign of the injected charge and the polymer chemistry. If there is a uniform electric field applied parallel to the chain axis and its strength is greater than a threshold value, these charged defects may or may not, depending on the polymer chemistry and substitution position, move along the chain for the new positions shown on the right-hand side of Fig. 1,.

In order to study the effect of the applied electric field on the mobility of such charged defects, we use the definition of mobility as velocity per unit applied field and estimate the velocity of the single defect (for single charged chain) or the centre of mass of both defects (for double charged chain) at different strengths of the applied field using a self-consistent molecular dynamics approach. The results obtained for PPV, 2-CN-PPV, 7-CN-PPV, 8-CN-PPV and DMeO-PPV are show in Fig. 2. There is a clear electric field dependence of the polaron mobility in PPV and CNPPV. In most cases, three different field-dependent regions are predicted. When the applied electric field is just above the field needed to move the injected charge and its associated deformation field along the chain, mobility increases as the field increases and becomes approximately constant for moderated field intensities. The field-independent region is followed by another region where polaron mobility increases sharply with the applied field until the coupling between the injected charge and the induced structural defect on the polymer backbone is broken. Our results for DMeOPPV are better described as field-independent. The dependence of intra-chain polaron-like mobility on the electric field predicted for PPV and its derivatives is quite different from the Poole-Frenkel form inferred from time-of-flight measurements in organic semiconductors [10].

Both positive and negative polaron-like transports can be realized along the PPV chains, but the maximum variation of their intra-chain mobility and the electric field range for polaron mobility depends on the number and sign of the injected charges. In contrast with PPV, only negative polarons are allowed to move along isolated chains of DMeO-PPV and 2-CN-PPV, whereas for 7$\mathrm{CN}-\mathrm{PPV}$ and 8-CN-PPV a polaron-type transport along the chains is predicted only for positive injected charges.

Comparing our quantitative estimate for the mobility of a single negative polaron along the DMeO-PPV chain $\left(0.57 \mathrm{~cm}^{2} \mathrm{~V}^{-1} \mathrm{~s}^{-1}\right)$ with the measurements by Hoofman et al. [3] for onedimensional intra-chain mobility of electrons on isolated polymer chains of poly(2-methoxy-5-[2'ethil-hexyloxy]-phenylene vinylene) $\left(0.5 \mathrm{~cm}^{2} \mathrm{~V}^{-1} \mathrm{~s}^{-1}\right)$, we find that our theoretical finding is in good agreement with experiment.

The effect of the conjugation length on the field-dependent polaron mobility along two PPV chains of 16 and 11 monomer units as a result of a single injected charge (electron or hole) is shown in Fig. 3. A decrease in the conjugation length leads to a larger change in mobility for both positive or negative polaron-like carriers and a larger electric field range for the mobility of such carriers, but the three regions of different field-dependent mobility are still predicted.

Finally, the effect of having a PPV chain with two conjugated segments perpendicular to each other [11] was investigated. It was found that mobility of polaron-like carriers along the entire chain crossing the twist between both segments is possible only if two charges of the same sign (either electrons or holes) are present in the chain, one in each segment. The polaron created when a single charge is injected into the chain is unable to cross the twist between both segments under the action of an external electric field as high as for a PPV chain with two conjugated segments 
with 11 and 5 monomer units in each. The mobilities predicted for positive and negative polaronlike carriers are field-independent and their maximum values are approximately $0.1 \mathrm{~cm}^{2} \mathrm{~V}^{-1} \mathrm{~s}^{-1}$. The presence of a conjugation break in the chain thus causes a decrease in the polaron mobility compared to a molecule without a conjugated break.

\section{Conclusions}

The injection of electrons or holes on isolated chains of PPV and its derivatives gives rise to charge-induced defects of polaronic-type on the polymer backbone. Both positive and negative polaron-like carriers can move along the PPV chains over some range of the applied electric field. Their intra-chain mobility initially increases as we increase the electric field and becomes approximately constant for the intermediate fields. This plateau is followed by a rapid increase in polaron mobility until the field is high enough to break the coupling between the injected charge and induced defect. The variation of the polaron mobility with the applied electric field is dependent of the number and sign of the injected charge and the conjugation length.

Not all PPV derivatives exhibit both positive and negative polaron-like transport. The existence of a polaron-like transport is a function of the type of substitute and substitution position. The polymer chemistry also affects the dependence of polaron mobility on the applied electric field. The good agreement between our calculated values for intra-chain mobility of electrons on isolated chains of a PPV derivative and experimental ones strongly suggests that self-consistent quantum molecular dynamics is a powerful computational method for investigating the dependence of the mobility of polaron-like carriers in conjugated polymers on applied electric fields, polymer chemistry and length This knowledge should prove invaluable in the development of mesoscopic models of electronic devices which will further enhance our understanding of their behaviour.

\section{Acknowledgement}

This work is part of the research projects POCTI/CTM/41574/2001 and CONC-REEQ/443/2001, approved by the Portuguese Foundation for Science and Technology (FCT) and supported by the European Community Fund FEDER. One of us (H.M.G.C.) is also indebted to FCT for financial support under PhD grant $n^{\circ}$. SFRH/BD/11231/2002.

\section{References}

1. J. H. Burroughes, D. D. C. Bradley, A. R. Brown, R. N. Marks, K. Mackay, R. H. Friend, P. L. Burns, A. B. Holmes, Nature 347, 539 (1990).

2. R. H. Friend, R. W. Gymer, A. B. Holmes, J. H. Burroughes, R. N. Marks, C. Taliani, D. D. C. Bradley, D. A. Dos Santos, J. L. Bredas, M. Logdlund, W. R. Salaneck, Nature 397, 121 (1999).

3. R. Hoofman, M. P. De Haas, L. D. A. Siebbeles, J. M. Warman, Nature 392, 54 (1998).

4. L. P. Candeias, F. C. Grozema, G. Padmanaban, S. Ramakrishnan, L. D. A. Siebbeles, J. M. Warman, J. Phys. Chem. B 107, 1554 (2003).

5. M. M. D. Ramos, Vacuum 64, 255 (2002).

6. H. M. G. Correia, M. M. D. Ramos, Materials Science \& Engineering C-Biomimetic and Supramolecular Systems 23, 773 (2003).

7. H. M. G. Correia, M. M. D. Ramos, Computational Materials Science 33, 218 (2005).

8. D. S. Wallace, Electron-lattice coupling in conjugated polymers, in "D. Phil. Thesis" (University of Oxford, 1989). 
9. D. S. Wallace, A. M. Stoneham, W. Hayes, A. J. Fisher, A. H. Harker, J. Phys.-Condes. Matter 3, 3879 (1991).

10. A. B. Walker, A. Kambili, S. J. Martin, J. Phys.-Condes. Matter 14, 9825 (2002).

11. H. M. G. Correia, H. M. C. Barbosa, M. M. D. Ramos, Journal of Non-Crystalline Solids 352 , 1691 (2006). 
Figure 1

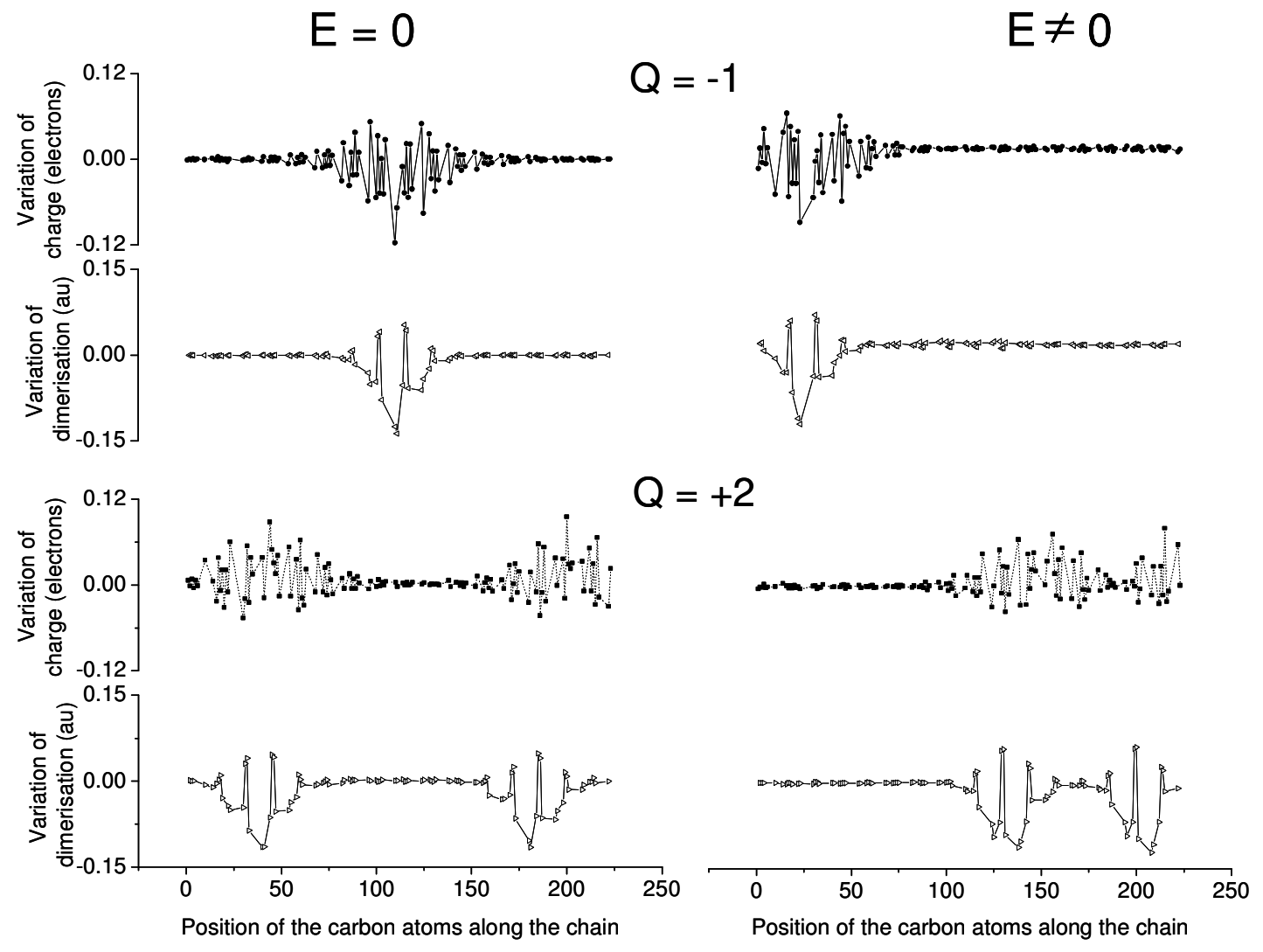


Figure 2
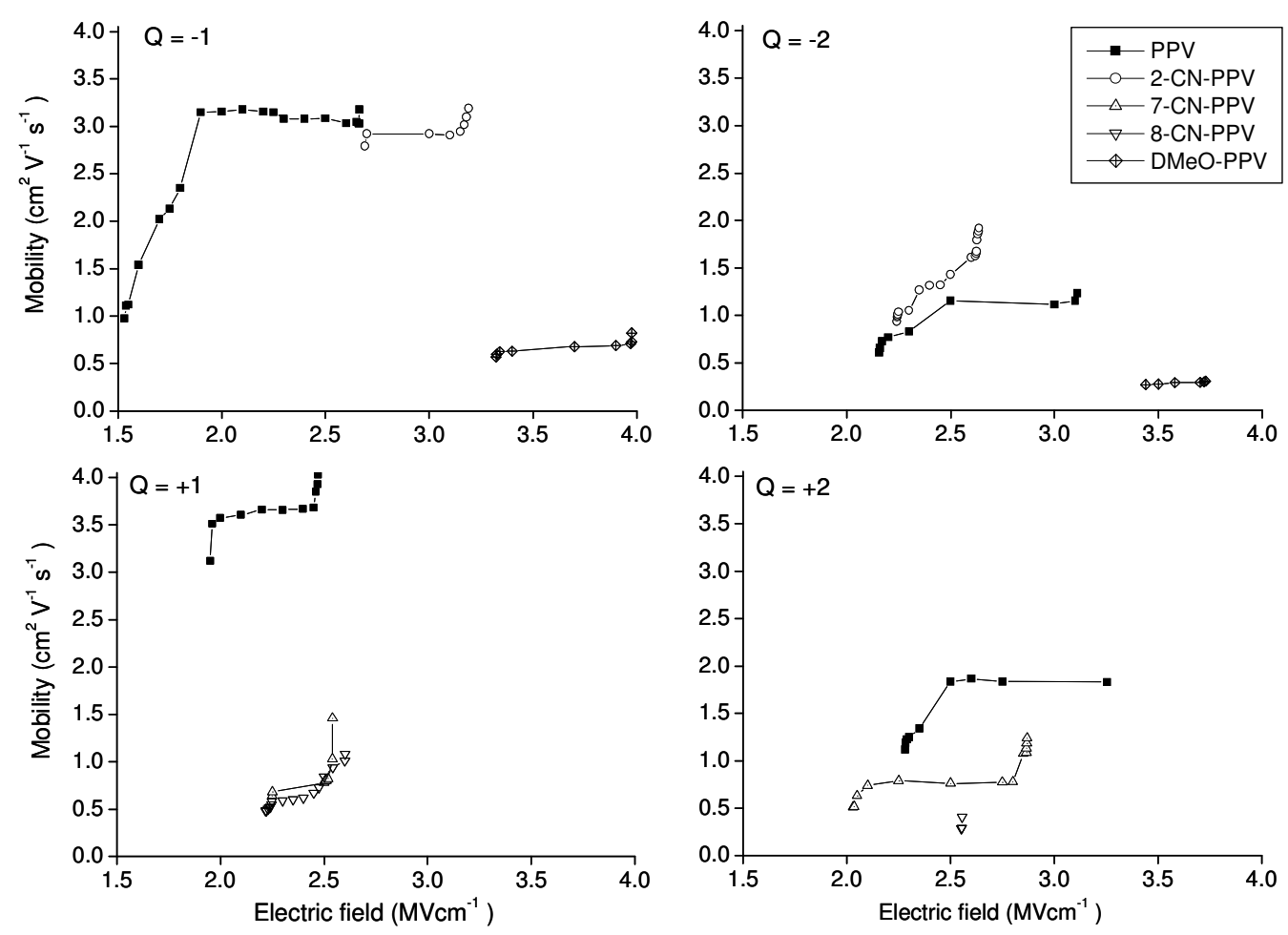
Figure 3

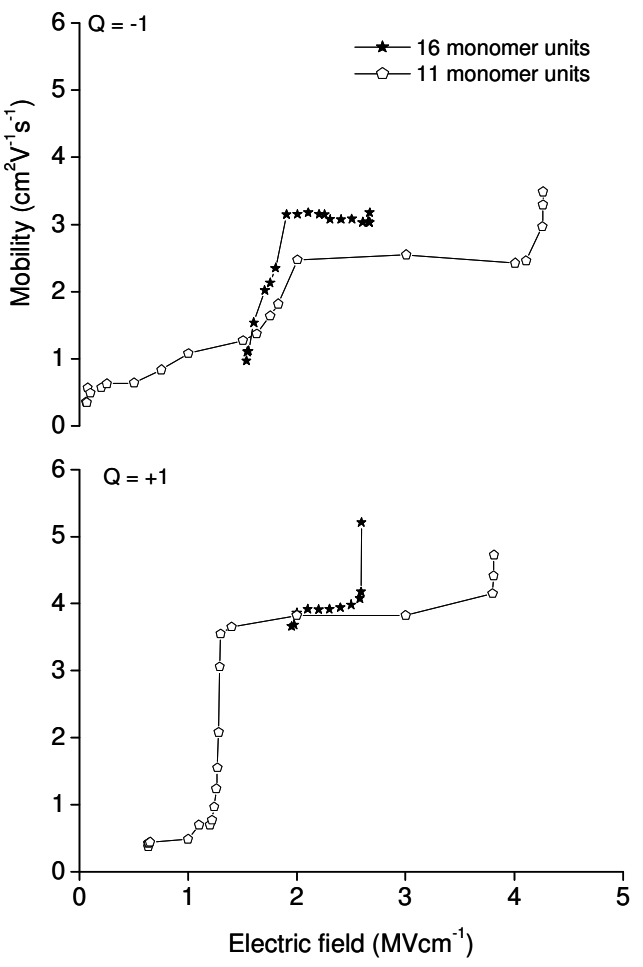


Figure legends

Figure 1 - The changes in the atomic charges and dimerisation pattern of carbon atoms of the PPV backbone caused by the injection of one electron $(\mathrm{Q}=-1)$ and two holes $(\mathrm{Q}=+2)$ into a chain with 16 monomer units, in the absence (left-hand site) and presence (right-hand site) of a strong enough electric field applied parallel to the chain axis to move the charge-induced defects to the chain end. The units used for charge and dimerisation are electrons $\left(1\right.$ electron $\left.=1.602 \times 10^{-19} \mathrm{C}\right)$ and atomic units $\left(1 \mathrm{au}=0.5291 \times 10^{-10} \mathrm{~m}\right)$, respectively.

Figure 2 - Calculated intra-chain mobility of charge-induced defects as a function of the applied electric field along the axis of a chain with 16 monomer units for PPV, 2-CN-PPV, 7-CN-PPV, 8$\mathrm{CN}-\mathrm{PPV}$ and DMeO-PPV, showing results for the movement of electrons $(\mathrm{Q}=-1$ and $\mathrm{Q}=-2)$ and holes $(\mathrm{Q}=+1$ and $\mathrm{Q}=+2)$ towards opposite chains ends.

Figure 3 - Calculated intra-chain mobility of positive and negative polaron-like carriers as a function of the applied electric field along the axis of two PPV chains with 16 and 11 monomer units, showing the results for the movement of a single injected electron $(\mathrm{Q}=-1)$ and hole $(\mathrm{Q}=+1)$ towards the same chain end. 